\title{
Correlation between peripapillary macular fiber layer thickness and visual acuity in patients with open-angle glaucoma
}

This article was published in the following Dove Press journal:

Clinical Ophthalmology

29 June 2010

Number of times this article has been viewed

\section{Kazuko Omodaka \\ Toru Nakazawa \\ Yu Yokoyama \\ Hiroshi Doi \\ Nobuo Fuse \\ Kohji Nishida}

Department of Ophthalmology, Tohoku University Graduate School of Medicine, Aoba-ku, Sendai, Miyagi, Japan
Correspondence: T Nakazawa Department of Ophthalmology, Tohoku University Graduate School of Medicine, I-I Seiryo-machi, Aoba-ku, Sendai, Miyagi 980-8574, Japan

Tel +8I 227177294

Fax +81227177298

Email ntoru@oph.med.tohoku.ac.jp
Purpose: To determine whether the best-corrected visual acuity (BCVA) is significantly correlated with the retinal nerve fiber layer thickness (RNFLT) in the different peripapillary areas in eyes with open-angle glaucoma (OAG).

Patients and methods: Two hundred twenty-nine eyes of 159 patients with OAG were studied. The overall RNFLT and the RNFLT in the superior (S), temporal (T), inferior (I), and nasal (N) quadrants were measured by Stratus optical coherence tomography (OCT). In addition, the temporal RNFLT was measured for three $30^{\circ}$ sectors with the mid-temporal (mT) sector at the three or nine o'clock position. The Spearman coefficient of correlation was calculated to determine whether a significant correlation existed between the RNFLT and the BCVA. Eyes with a BCVA $<20 / 20$ were classified as having decreased BCVA.

Results: There were significant correlations between the BCVA and the overall RNFLT, and also between the BCVA and the RNFLT in the T, I, and S quadrants. The coefficient correlation between the BCVA and RNFLT was the highest in the mT sector $(\mathrm{r}=-0.40, P<0.0001)$. Forty-eight eyes $(21.1 \%)$ were classified as having decreased BCVA. The cut-off value of the $\mathrm{mT}$ sector for eyes with decreased VA was $39.0 \mu \mathrm{m}$.

Conclusion: The highest correlation between the BCVA and the RNFLT in the mT sector was most likely due to the location of the maculopapillary bundle in this region of the optic disc. Our findings indicate that when the RNFLT in the $\mathrm{mT}$ sector is reduced to $40 \mu \mathrm{m}$, glaucoma treatment should be reconsidered.

Keywords: retinal nerve fiber layer, low vision, peripapillary macular bundle

\section{Introduction}

Glaucoma affects over 70 million people worldwide ${ }^{1,2}$ and is the second most frequent cause of blindness. ${ }^{1}$ A major risk factor of glaucoma is increased age, and the lengthening life span worldwide has increased the number of individuals with glaucoma and blindness. ${ }^{3}$ Generally, the central visual fields and visual acuity (VA) are preserved until the late stages of glaucoma. However there are patients whose VA is decreased even in the early stages of glaucoma. ${ }^{4,5}$ Because of the high density of RGCs in the fovea, the retinal nerve fiber layer (RNFL) from the foveal area is thicker than that from the peripheral area of the retina. However, it is estimated that a loss of $20 \%$ of the RGC is necessary to detect a $5 \mathrm{~dB}$ decrease in mean deviation (MD) of the standard automated perimetry (SAP). ${ }^{6}$ Thus, examination by SAP has been reported to be not adequate for detecting early stage glaucoma. ${ }^{7}$ Instead, optical coherence tomography (OCT) and Heidelberg Retina Tomograph-II (HRT-II) or their joint use has been recommended for detecting early stage glaucoma. ${ }^{8-10}$ 
OCT is a noninvasive, noncontact, objective method of obtaining a cross-sectional image of the retina with resolution of approximately $10 \mu \mathrm{m}$. The OCT findings are repeatable with a coefficient of variation ranging from $3.8 \%$ to $5.2 \%,{ }^{11}$ and measurements of the RNFL thickness (RNFLT) can assist in the diagnosis of early glaucoma. It has been shown that the RNFLT is highly correlated with the mean deviations (MDs) of the Humphrey field analyzer (HFA). ${ }^{12}$ Thus, the evaluation of RNFLT by OCT has emerged as an important method for pre-perimetric diagnosis of glaucoma and may help not only in an accurate and early diagnosis of glaucoma, ${ }^{10,13}$ but also as a method to follow glaucomatous progression.

Because there are a few reports on cases with a central scotoma and decreased VA due to glaucoma, ${ }^{4,5,14}$ we first surveyed the number of glaucoma patients with decreased BCVA in our outpatient glaucoma clinic. We investigated whether the OCT-determined RNFLT would help in the detection of these glaucoma patients with decreased BCVA. We were specifically interested in which regions of the RNFLT were best correlated with the BCVA in these patients with glaucoma. We shall show that the thickness of the temporal RNFLT of the optic disc was more highly correlated with the BCVA, and the middle temporal (mT) 30 area which included maculopapillary bundle had the highest coefficient of correlation.

\section{Subjects and methods Patient eligibility criteria}

Patients were included if they: (1) were diagnosed with open-angle glaucoma (OAG) including primary open-angle glaucoma (POAG) and normal tension glaucoma (NTG); (2) had refractive errors (spherical equivalent) of $<+3.0$ diopters (D) and $>-8.00 \mathrm{D}$; and (3) had glaucomatous visual field meeting the Anderson-Pattela classification. ${ }^{15}$ Patients were excluded if they had any of the following during the follow-up: (1) development of any ocular disease; (2) systemic diseases affecting the visual fields; (3) intraocular surgery; and (4) cataract progression.

The baseline clinical parameters including age, gender, and refractive error for each patient were recorded. The baseline best-corrected visual acuity (BCVA) was measured with a standard Japanese decimal visual acuity chart and converted to the base 10 logarithm of the minimum angle of resolution (logMAR) for the statistical analyses. The IOPs were measured by Goldmann applanation tonometry at the time of the initial diagnosis of OAG without any medications for the glaucoma.

This study followed the Tenets of the Declaration of Helsinki and approval for the analysis of the retrospective data was obtained from the Institutional Review Board of the Tohoku Graduate School of Medicine.

\section{Classification of disc appearance}

The optic discs were divided into myopic glaucomatous disc (MY) or non-MY types. In the MY disc type, the optic disc had temporal peripapillary atrophy with temporal cupping, and was tilted slightly temporally. Two glaucoma specialists classified the disc types independently, and if their classification did not agree and the optic disc was difficult to classify, the case was excluded from the statistical analyses. The percentage of cases that were excluded was $18.8 \%$. Patients with abnormal disc appearances, including large disc, small disc, and lower conus were also excluded.

\section{Visual field analyses}

The mean deviation (MD) values were obtained by the Swedish interactive threshold algorithm (SITA)-standard strategy of the 30-2 program of the Humphrey field analyzer (Carl Zeiss Meditec, Dublin, CA, USA). The MD values were obtained from reliable visual fields, ie, $<20 \%$ fixation errors, $<33 \%$ false-positives, and $<33 \%$ false-negatives.

\section{Regional RNFLT measurements with Stratus OCT}

The RNFLT was measured with the Stratus OCT instrument software version 4.0.1, (Carl Zeiss Meditec, Inc.). ${ }^{16,17}$ The fast RNFL protocol (consisting of three circular scans $(3.46 \mathrm{~mm}$ in diameter) centered on the optic disc) was used. The overall RNFLT covering the entire circumference of the optic disc was determined by the embedded program of the Stratus OCT. Similarly, the RNFLT in the four quadrants, the superior (S; $315^{\circ}$ to $45^{\circ}$ ), temporal ( $\mathrm{T} ; 225^{\circ}$ to $315^{\circ}$ in the right eye, $45^{\circ}$ to $135^{\circ}$ in the left eye), inferior (I; $135^{\circ}$ to $225^{\circ}$ ), and nasal (N; $45^{\circ}$ to $135^{\circ}$ in the right eye, $225^{\circ}$ to $315^{\circ}$ in the left), were measured. The temporal RNFL of the optic disc was subdivided into three $30^{\circ}$ sectors with the mid-temporal (mT) sector centered at $9 \mathrm{o}$ 'clock for the right eye or at 3 o'clock for the left eye. The segmentation algorithms occasionally failed, especially with glaucomatous eyes with poor quality OCT images. Therefore, images with signal intensity $<6$, or were poor with questionable segmentation were excluded.

\section{Statistical analyses}

Analysis of variance for continuous data (Kruskal-Wallis test: ANOVA followed by Scheffe post hoc test) was used to determine the significance of differences in the RNFLT in the four quadrants. The significance level was set $P<0.05$. 
Fisher's exact test was used for statistical analysis of the categorical data. Spearman's correlation analysis was used to determine the correlations between the BCVA and the IOP without medication and with the RNFLT in the different regions of the optic disc. The receiver operating characteristic (ROC) curve for the RNFLT in different areas, the areas under the curve (AUC), and the cut-off values for eyes with reduced BCVA $(<20 / 20)$ due to glaucoma were calculated with the JMP software version 8.0.1, (SAS Institute Japan Inc., Tokyo, Japan). The data are expressed as the means \pm standard deviations (SDs).

\section{Results}

Two hundred twenty-nine eyes (men 46\%, women 54\%) were studied. The average age of the patients with OAG was $56.7 \pm 14.5 \mathrm{yrs}$, the refractive error (spherical equivalent) was $-3.1 \pm 2.7$ diopters (D), the MD was $-9.2 \pm 6.8$ $\mathrm{dB}$ (median: $-7.7 \mathrm{~dB}$ ), the BCVA was $0.0062 \pm 0.2$ $\log$ MAR units, and the baseline IOP was $18.4 \pm 7.0 \mathrm{mmHg}$ (Table 1).

The average overall RNFLT was $74.7 \pm 17.5 \mu \mathrm{m}$, the $\mathrm{S}$ quadrant was $94.4 \pm 27.5 \mu \mathrm{m}$, the $\mathrm{T}$ quadrant was $57.9 \pm 18.3 \mu \mathrm{m}$, the I quadrant was $83.1 \pm 27.0 \mu \mathrm{m}$, and the $\mathrm{N}$ quadrant was $62.8 \pm 15.5 \mu \mathrm{m}$. For the temporal RNFLT, the superior temporal (sT) sector was $66.1 \pm 25.0 \mu \mathrm{m}, \mathrm{mT}$ sector was $52.4 \pm 16.8 \mu \mathrm{m}$, and the inferior $\mathrm{T}$ (iT) sector was $55.2 \pm 21.5 \mu \mathrm{m}$ (Table 2).

Forty-eight of the 229 eyes (21.1\%) were placed in the decreased BCVA group. There were weak but significant correlations between the BCVA and the IOP $(r=0.201$, $P=0.024)$, and between the BCVA and the overall RNFLT $(\mathrm{r}=-0.289, P<0.0001)$. The correlations between the BCVA and the RNFLT of the T quadrant $(\mathrm{r}=-0.368, P<0.0001)$, the $\mathrm{S}$ quadrant $(\mathrm{r}=-0.289, P=0.001)$, and the I quadrant

Table I Demographic data of the patients with or without glaucomatous myopic disc

\begin{tabular}{llll}
\hline & $\begin{array}{l}\text { MY } \\
(\mathbf{n}=\mathbf{1 2 9})\end{array}$ & $\begin{array}{l}\text { non-MY } \\
(\mathbf{n}=\mathbf{1 0 0})\end{array}$ & $\begin{array}{l}\text { Total } \\
(\mathbf{n}=\mathbf{2 2 9})\end{array}$ \\
\hline Sex & & & \\
$\quad$ Male, $\mathrm{n}(\%)$ & $65(50.4 \%)$ & $40(40.0 \%)$ & $105(45.8 \%)$ \\
$\quad$ Female, $\mathrm{n}(\%)$ & $64(49.6 \%)$ & $60(60.0 \%)$ & $124(54.1 \%)$ \\
$\quad$ Age, yrs (mean \pm SD) & $51.1 \pm 14.1^{*}$ & $64.0 \pm 11.5$ & $56.7 \pm 14.5$ \\
SE (D) & $-4.3 \pm 2.5^{*}$ & $-1.5 \pm 2.6$ & $-3.1 \pm 2.7$ \\
MD (dB) & $-10.3 \pm 7.4^{*}$ & $-7.8 \pm 5.7$ & $-9.2 \pm 6.8$ \\
logMAR & $0.0128 \pm 0.2$ & $-0.0022 \pm 0.2$ & $0.0062 \pm 0.2$ \\
IOP (mmHg) & $19.0 \pm 7.2$ & $18.0 \pm 6.6$ & $18.4 \pm 7.0$ \\
\hline
\end{tabular}

Notes: The data showed the means \pm standard deviation (SD). $* P<0.05$, compared with that of non-MY.

Abbreviations: SE, spherical equivalent, MD, mean deviation of Humphrey fields analyzer; IOP, intraocular pressure.
Table 2 Average of regional RNFLTs

\begin{tabular}{ll}
\hline Region & RNFLT $(\mu \mathrm{m})$ \\
\hline Average & $74.7 \pm 17.5$ \\
Superior & $94.4 \pm 27.5$ \\
Temporal & $57.9 \pm 18.3$ \\
Inferior & $83.1 \pm 27.0$ \\
Nasal & $62.8 \pm 15.5$ \\
sT & $66.1 \pm 25.0$ \\
mT & $52.4 \pm 16.8$ \\
iT & $55.2 \pm 21.5$ \\
\hline
\end{tabular}

Abbreviations: Avg, average of all RNFLT;S, superior;T,temporal; I, inferior; $\mathrm{N}$, nasal. Quadrant temporal RNFLT was further divided into 3 regions (30 degree each), sT, superiotemporal; mT, midtemporal; iT, inferotemporal; RNFLT, retinal nerve fiber layer thickness.

( $\mathrm{r}=-0.202, P<0.0001)$ were all significant. However that for the $\mathrm{N}$ quadrant was not significant $(\mathrm{r}=-0.095 ; P=0.051$; Figure 1B). For the temporal area of optic disc, there were significant correlations between the BCVA and the $\mathrm{mT}$ sector ( $\mathrm{r}=-0.396, P<0.0001$, Figure $1 \mathrm{~A})$, the iT sector ( $\mathrm{r}=-0.345, P<0.0001)$, and the sT sector $(\mathrm{r}=-0.269$, $P<0.0001$; Figure 1). The coefficient of correlation was the highest for the $\mathrm{mT}$ sector.

Logistic regression analysis with the AUC of the ROC showed that AUC of the overall RNFLT was 0.743 (Figure 2). For the different quadrants, the AUC in the T quadrant was the highest at 0.777 , and then 0.700 for the I quadrant, 0.668 for the $\mathrm{S}$ quadrant, and 0.612 for the $\mathrm{N}$ quadrant. In addition, the $\mathrm{mT}$ sector had the highest AUC at 0.784 , and the next was 0.745 in the iT sector and 0.726 in the sT sector (Figure 2 ). The cut-off values of the overall RNFLT for eyes with VA $<20 / 20$ was $61.6 \mu \mathrm{m}$, and $53.0 \mu \mathrm{m}$ for the T quadrant, and $39.0 \mu \mathrm{m}$ for the $\mathrm{mT}$ sector (Figure 2A). The sensitivity in the mT sector was 0.62 and the specificity was 0.86 .

Among the two disc types, MY and non-MY, patients with the MY disc type were significantly younger $(P<0.0001)$, more myopic $(P<0.0001)$, and had worse $\operatorname{MDs}(P=0.0142)$ than those with the other disc type (Table 1). However, the differences in the gender, BCVA, and IOP between the MY disc type and non-MY disc type were not significant (Table 1). When the criterion BCVA was set at $<0.3(<20 / 60)$ for selecting patients with decreased VA, there were more patients with decreased VA in the MY disc type than in the non-MY disc types $(P=0.0425$; Figure 3$)$.

A representative glaucoma patient with decreased VA (20/100) with the MY disc (Figure 4A, C, E, G) and a normal case $(V A=20 / 20)$ with the MY disc (Figure 4B, D, F, H) are shown in Figure 4. The glaucoma patient was a 63-year-old man who had NTG with an HFA -7.38 dB mean deviation. His refractive error was $-4.75 \mathrm{D}$ and IOP was $12 \mathrm{mmHg}$. 
A
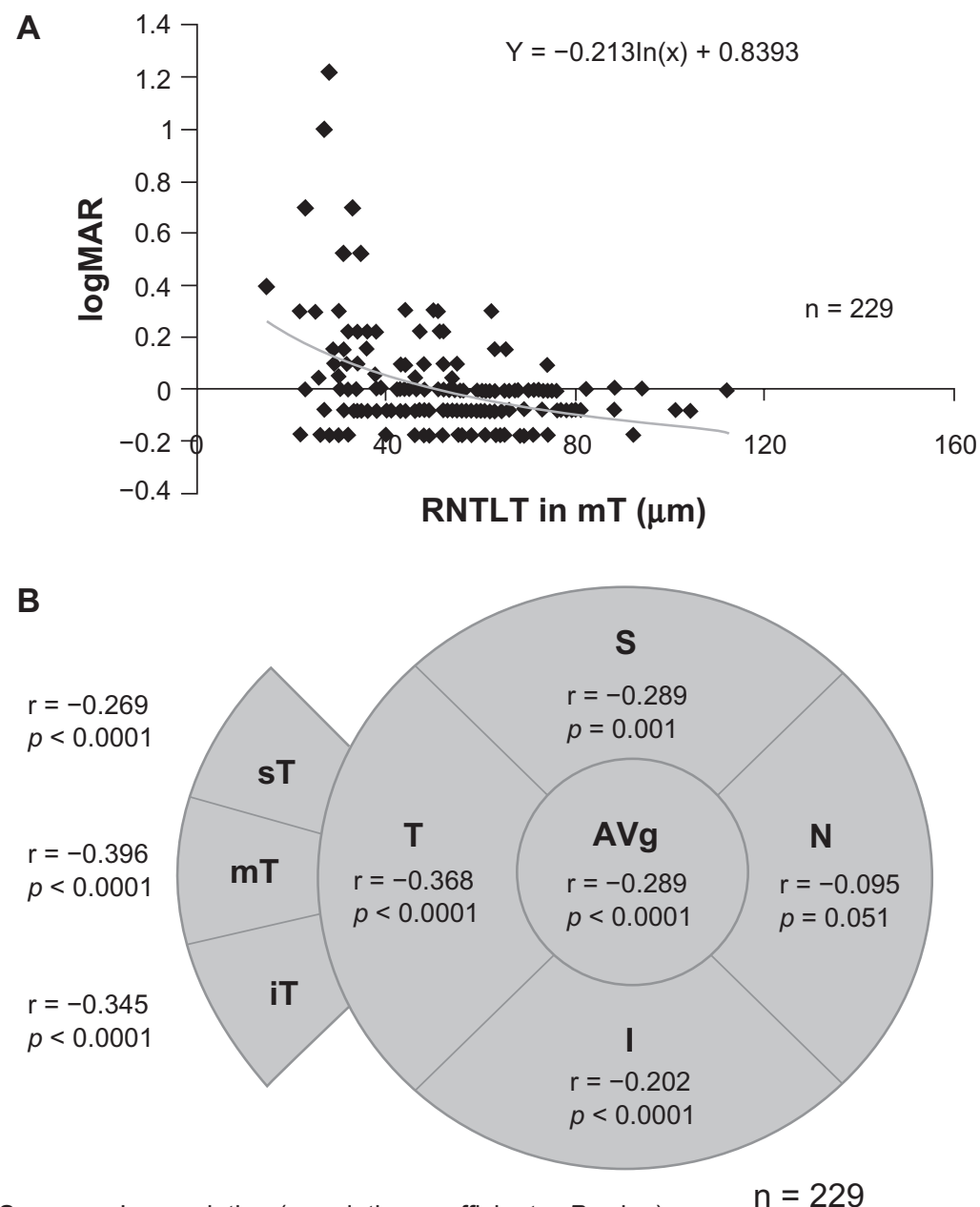

Spearman's correlation (correlation coefficient $r, P$ value)

Figure I Coefficient of correlations ( $r$ ) between the BCVA and the RNFLT in different regions of the optic disc. A) Scatterplot graph showing the association between logMAR BCVA and the RNFLT in the mT sector. B) Diagram illustrating the relationship between the visual field location and the BCVA/RNFLT correlation data. Upper line, correlation coefficient value $(r)$; and lower line, $P$ value. Statistical analysis was performed by Spearman's correlation. Average overall RNFLT; S, superior quadrant; $\mathrm{T}$, temporal quadrant; I, inferior quadrant; and N, nasal quadrant. The temporal RNFLT was divided into 3 sectors of $30^{\circ}$; sT, superiotemporal sector; mT, midtemporal sector; iT, inferotemporal sector.

The normal patient was a 66-years-old man with a refractive error of $-5.0 \mathrm{D}$ and an IOP of $12 \mathrm{mmHg}$.

The glaucoma patients with decreased VA had a thinner RNFLT in the $\mathrm{mT}$ sector (Figure 4C, D) and a dark fluorescence at the optic disc indicating a lower blood perfusion (filling defect) in the optic disc which was demonstrated by fluorescent angiography (Figure 4E), while the filling defect was not detected in the normal patients with the myopic disc (Figure 4F).

\section{Discussion}

Our results showed that there was a weak but significant correlation between the BCVA and the overall RNFLT obtained by Stratus OCT. Analyses of the different regions of the RNFL showed that the temporal area had the highest correlation to the BCVA among the four quadrants, and the correlation was highest for the $\mathrm{mT}$ ( 3 or 9 o'clock) sector of the optic disc. This is reasonable because this region includes the peripapillary macular bundle.

Among our patients, there were $21.1 \%$ with BCVA $<20 / 20$. The ROC analyses showed the same trends as those obtained for the RNFLT. The RNFLT cut-off values for the glaucomatous eyes with BCVA $<20 / 20$ was $39.0 \mu \mathrm{m}$. However, there are limitations to this cut-off value because some patients with RNFLT $<39 \mu \mathrm{m}$ still had good vision and some with RNFLT $>39 \mu \mathrm{m}$ had poor vision. Our conclusion was reached by statistical analyses of our data, and we need to be careful in interpreting the data because the structure and the function were not always correlated.

Although glaucoma is generally considered to be a disease in which the VA is relatively good, our data showed that the percentage of OAG patients with decreased VA $(<20 / 20)$ 
A
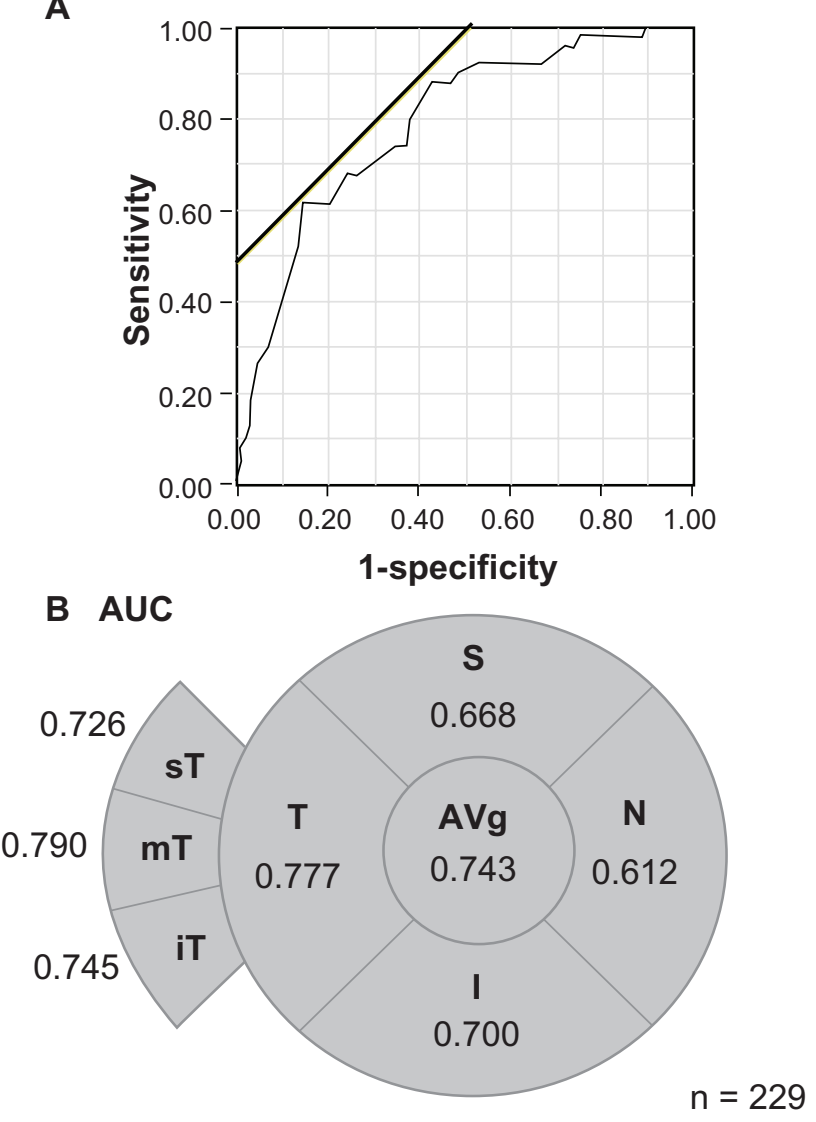

Figure 2 Visual field regional values of area under curve (AUC) of the receiver operating characteristics between RNFLT and visual acuity. A) ROC plot shows the association between logMAR BCVA and RNFLT in the $\mathrm{mT}$ sectors. The oblique line is the cut-off values. B) AUC data displayed as a function of visual field location. Average overall RNFLT; S, superior quadrant; T, temporal quadrant; I, inferior quadrant; and N, nasal quadrant. The temporal RNFLT was divided into 3 sectors of $30^{\circ}$; sT, superiotemporal sector; mT, midtemporal sector; and iT, inferotemporal sector.

was not insignificant (21.1\%). In these patients, the highest correlation between the RNFLT and the BCVA was for the $\mathrm{mT}$ sector which included the peripapillary macular bundle. This is in agreement with the report that an examination of the peripapillary RNFLT will provide a better chance of detecting early glaucoma than that of the T quadrant of the RNFLT. ${ }^{18}$ The peripapillary RNFLT is also significantly correlated with the severity of neuro-ophthalmological diseases such as chiasmal tumors ${ }^{19}$, and is linearly correlated with VA in the patients with optic neuritis. ${ }^{20}$ Thus, the peripapillary RNFLT measured by Stratus OCT is a better objective parameter to assess axonal degenerative ocular diseases not only for glaucoma but also for neuro-ophthalmological diseases.

To interpret the Stratus OCT-determined sectorial RNFLT, attention must be paid to the limitation of this technique, viz., glial proliferation and the reproducibility. After axonal degeneration, glial cells tend to proliferate ${ }^{21}$ into the nerve fiber layer. Because OCT measures the combined axon fibers and glial cells, the RNFLT is not an accurate measure of the real number of RGC axons at the late stage of glaucoma.

The standard deviation of the thickness of the quadrant sector $\left(90^{\circ}\right)$ of the RNFLT ranges from 6 to $16 \mu \mathrm{m}$ which was less than that in the narrower sectors $\left(30^{\circ}\right)$ of the RNFLT where it was $20 \mu \mathrm{m} .{ }^{11}$ Thus, these variances must be considered when the RNFLT of the mT region is used to assess the degree of damage to the axons in the peripapillary bundle (Figure 1). If the reproducibility of the narrower RNFLT can be improved, eg, by a quicker scan or by more accurate tracking of the eye, measurements of the RNFLT in the mT sector would be more valuable for assessing the visual function objectively.

Carroll and Forbes ${ }^{4}$ postulated that glaucoma-associated central visual field defects may be related to myopia. On the other hand, Pickett et al reported that among ten glaucoma patients with early loss of the central visual acuity, only three were myopic. ${ }^{5}$ Ivers et al ${ }^{14}$ studied 3654 subjects, and

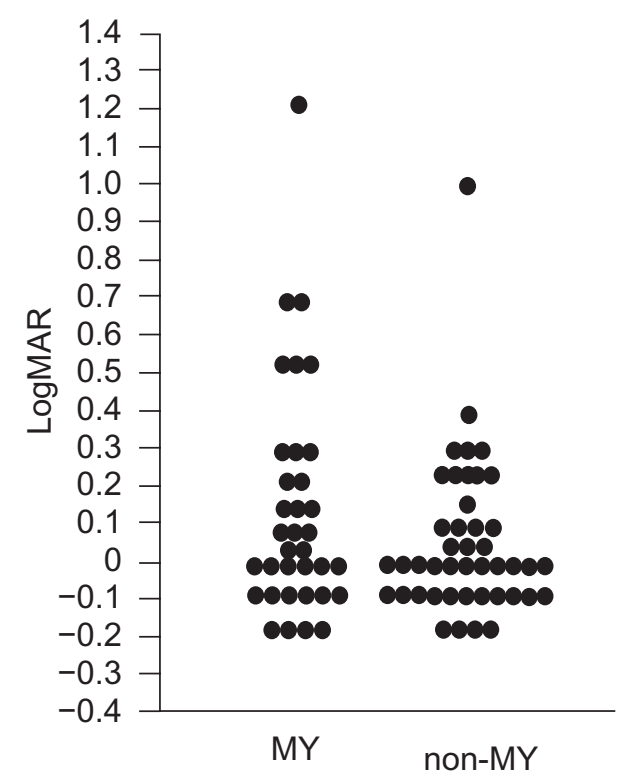

\begin{tabular}{cccc}
\hline & MY & non-MY & Total \\
\hline$<=0.3$ & $8^{*}$ & 1 & 9 \\
$>0.3$ & 121 & 99 & 220 \\
\hline Total & 129 & 100 & 229 \\
\hline
\end{tabular}

${ }^{*} P=0.0425$ : MY v.s. non-MY $(2 \times 2$ fisher exact test $)$

Figure 3 LogMAR best corrected visual acuity (BCVA) between the patients with the MY disc type and the non-MY disc type. A) Dot blot shows the distribution of logMAR BCVA in the MY and non-MY disc types. B) The Fisher exact probability test based on the tabulated data indicates that there are more patients with low BCVA ( 0.3 or less) in the MY disc types than in the non-MY disc types. 

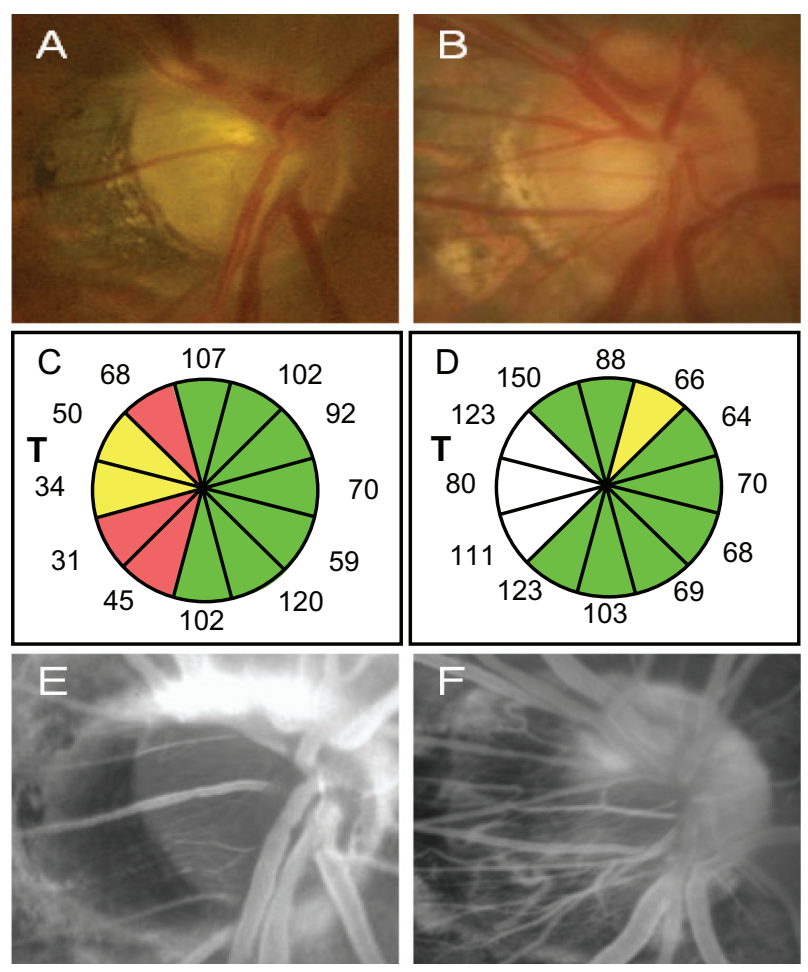

Figure 4 Representative glaucoma patient with decreased VA with the MY disc (Fig. 4A, C, E, G) and normal case with the MY disc (Fig. 4B, D, F, H). A, B) The fundus photographs of optic disc. C, D) The OCT determined- 12 sectored RNFLT. T: temporal. E, F) Fluorescein angiograms of the optic disc at the late stage (6 mins).

reported that the BCVA had the same or better sensitivity and specificity for detecting glaucoma than the IOP. Our results showed that the number of glaucoma patients with severely decreased VA ( 0.3 or less) was significantly higher in the eyes with the MY disc type. In addition, patients with decreased VA in the MY disc type had thinner RNFLT in the $\mathrm{mT}$ section and lower blood perfusion (filling defect). A filling defect on the surface of optic disc is specific for glaucoma, ${ }^{22,23}$ however, it must still be confirmed whether the vascular change is just associated or plays a causative role in the decreased VA in the patients with MY disc type.

Asians $^{24}$ are characterized by a higher prevalence of myopia than individuals from Western countries, ${ }^{25,26}$ and our data suggest that there is a higher risk among Asians for a decrease in the VA due to glaucoma than among individuals in the Western countries.

In conclusion, our findings suggest that the RNFLT, especially in the temporal area, would explain the decrease in the BCVA in glaucoma patients, and if the thickness is close to $40 \mu \mathrm{m}$, there is a significant risk for decreasing VA due to glaucoma.

\section{Acknowledgment}

Toru Nakazawa and Kazuko Omodaka equally contributed to this study. The authors thank Prof Duco Hamasaki for editing this manuscript.

\section{Disclosure}

The authors report no conflicts of interest in this work.

\section{Reference}

1. Quigley HA. Number of people with glaucoma worldwide. Br J Ophthalmol. 1996;80:389-393.

2. Resnikoff S, Pascolini D, Etya'ale D, et al. Global data on visual impairment in the year 2002. Bull World Health Organ. 2004;82: $844-851$.

3. Le A, Mukesh BN, McCarty CA, Taylor HR. Risk factors associated with the incidence of open-angle glaucoma: the visual impairment project. Invest Ophthalmol Vis Sci. 2003;44:3783-3789.

4. Carroll FD, Forbes M. Centrocaecal scotomas due to glaucoma. Trans Am Acad Ophthalmol Otolaryngol. 1968;72:643-648.

5. Pickett JE, Terry SA, O'Connor PS, O'Hara M. Early loss of central visual acuity in glaucoma. Ophthalmology. 1985;92:891-896.

6. Quigley HA, Dunkelberger GR, Green WR. Retinal ganglion cell atrophy correlated with automated perimetry in human eyes with glaucoma. Am J Ophthalmol. 1989;107:453-464.

7. Zeyen TG, Caprioli J. Progression of disc and field damage in early glaucoma. Arch Ophthalmol. 1993;111:62-65.

8. Quigley HA, Katz J, Derick RJ, Gilbert D, Sommer A. An evaluation of optic disc and nerve fiber layer examinations in monitoring progression of early glaucoma damage. Ophthalmology. 1992;99:19-28.

9. Sommer A, Katz J, Quigley HA, et al. Clinically detectable nerve fiber atrophy precedes the onset of glaucomatous field loss. Arch Ophthalmol. 1991;109:77-83.

10. Badala F, Nouri-Mahdavi K, Raoof DA, et al. Optic disk and nerve fiber layer imaging to detect glaucoma. Am J Ophthalmol. 2007; 144:724-732.

11. Budenz DL, Fredette MJ, Feuer WJ, Anderson DR. Reproducibility of peripapillary retinal nerve fiber thickness measurements with stratus OCT in glaucomatous eyes. Ophthalmology. 2008;115: 661-666 e664.

12. Kanamori A, Nakamura M, Escano MF, et al. Evaluation of the glaucomatous damage on retinal nerve fiber layer thickness measured by optical coherence tomography. Am J Ophthalmol. 2003;135:513-520.

13. Mardin CY, Junemann AG. The diagnostic value of optic nerve imaging in early glaucoma. Curr Opin Ophthalmol. 2001;12:100-104.

14. Ivers RQ, Optom B, Macaskill P, Cumming RG, Mitchell P. Sensitivity and specificity of tests to detect eye disease in an older population. Ophthalmology. 2001;108:968-975.

15. Anderson DR, Patella VM: Automated Static Perimetry (ed 2nd), Mosby, 1999.

16. Huang D, Swanson EA, Lin CP, et al. Optical coherence tomography. Science. 1991;254:1178-1181.

17. Schuman JS, Hee MR, Arya AV, et al. Optical coherence tomography: a new tool for glaucoma diagnosis. Curr Opin Ophthalmol. 1995;6: 89-95.

18. Leung CK, Chan WM, Yung WH, et al. Comparison of macular and peripapillary measurements for the detection of glaucoma: an optical coherence tomography study. Ophthalmology. 2005;112:391-400.

19. Kanamori A, Nakamura M, Matsui N, et al. Optical coherence tomography detects characteristic retinal nerve fiber layer thickness corresponding to band atrophy of the optic discs. Ophthalmology. 2004; 111:2278-2283. 
20. Naismith RT, Tutlam NT, Xu J, et al. Optical coherence tomography is less sensitive than visual evoked potentials in optic neuritis. Neurology. 2009;73:46-52.

21. Radius RL, Anderson DR. The histology of retinal nerve fiber layer bundles and bundle defects. Arch Ophthalmol. 1979;97:948-950.

22. Schwartz B. Circulatory defects of the optic disk and retina in ocular hypertension and high pressure open-angle glaucoma. Surv Ophthalmol. 1994;38 Suppl:S23-S34.

23. Talusan E, Schwartz B. Specificity of fluorescein angiographic defects of the optic disc in glaucoma. Arch Ophthalmol. 1977;95:2166-2175.
24. Sawada A, Tomidokoro A, Araie M, Iwase A, Yamamoto T. Refractive errors in an elderly Japanese population: the Tajimi study. Ophthalmology. 2008;115:363-370 e363.

25. Kempen JH, Mitchell P, Lee KE, et al. The prevalence of refractive errors among adults in the United States, Western Europe, and Australia. Arch Ophthalmol. 2004;122:495-505.

26. Sperduto RD, Seigel D, Roberts J, Rowland M. Prevalence of myopia in the United States. Arch Ophthalmol. 1983;101:405-407.
Clinical Ophthalmology

\section{Publish your work in this journal}

Clinical Ophthalmology is an international, peer-reviewed journal covering all subspecialties within ophthalmology. Key topics include: Optometry; Visual science; Pharmacology and drug therapy in eye diseases; Basic Sciences; Primary and Secondary eye care; Patient Safety and Quality of Care Improvements. This journal is indexed on

Submit your manuscript here: http://www.dovepress.com/clinical-ophthalmology-journal

\section{Dovepress}

PubMed Central and CAS, and is the official journal of The Society of Clinical Ophthalmology (SCO). The manuscript management system is completely online and includes a very quick and fair peer-review system, which is all easy to use. Visit http://www.dovepress.com/ testimonials.php to read real quotes from published authors. 